Revista Brasileira de Agricultura Irrigada v.10, nº.2, p. 576 - 585, 2016

ISSN 1982-7679 (On-line)

Fortaleza, CE, INOVAGRI - http://www.inovagri.org.br

DOI: $10.7127 /$ rbai.v10n200407

Protocolo 407.16 - 26/02/2016 Aprovado em 11/04/2016

\title{
MÉTODOS EMPÍRICOS PARA ESTIMATIVA DA EVAPOTRANSPIRAÇÃO DE REFERÊNCIA NO ESTADO DO RIO DE JANEIRO
}

\author{
Natan Didoné Vallory ${ }^{1}$, Rafael Esteves Dohler ${ }^{2}$, Roberto Avelino Cecílio ${ }^{3}$, Sidney Sára \\ Zanetti $^{4}$
}

\section{RESUMO}

O uso da água de forma racional tem se tornado cada vez mais importante nos últimos anos. A evapotranspiração é uma importante variável do ciclo hidrológico, no qual o seu conhecimento é essencial para o manejo de irrigação. Objetivou-se avaliar o desempenho dos métodos de Priestley-Taylor, Hargreaves-Samani, Thornthwaite e Camargo, em comparação com o método de Penman-Monteith, na estimação da evapotranspiração de referência em três municípios do Estado do Rio de Janeiro. Foram utilizadas variáveis climáticas referentes ao período de 01 de janeiro de 2011 a 31 de dezembro de 2013, das estações meteorológicas automáticas do INMET, nas cidades de Paraty, Petrópolis e Campos dos Goytacazes. Avaliaram-se os métodos nas escalas diária e mensal. Para a análise comparativa entre os resultados de cada método com o padrão, utilizou-se a regressão linear simples, o coeficiente de correlação de Pearson (r), o índice de concordância Willmott (d), e o índice de confiança de Camargo \& Sentelhas (c). Na escalas estudadas, os métodos de Priestley-Taylor e Hargreaves-Samani apresentaram desempenho superior aos demais métodos avaliados. O método de Priestley-Taylor mostrou-se mais confiável na estimação da evapotranspiração de referência em Paraty e Campos dos Goytacazes, e o método de Hargreaves-Samani para Petrópolis.

Palavras-chave: irrigação, Penman-Monteith, variáveis climáticas

\section{METHODS FOR EMPIRICAL ESTIMATE OF EVAPOTRANSPIRATION REFERENCE IN RIO DE JANEIRO STATE}

\footnotetext{
${ }^{1}$ Graduando em Engenharia Florestal, Universidade Federal do Espírito Santo, Campus Alegre, Alto Universitário, s/no, Guararema, Alegre - ES, CEP 29500-000, e-mail: natanvallory@gmail.com.

${ }^{2}$ Engenheiro Florestal, Mestrando em Ciências Florestais, Departamento de Ciências Florestais e da Madeira, Universidade Federal do Espírito Santo, Jerônimo Monteiro - ES, email: rafaelesteves_123@ hotmail.com (autor correspondente).

${ }^{3}$ Engenheiro Agrícola, D.Sc em Eng. Agrícola, Professor Associado, Departamento de Ciências Florestais e da Madeira, Universidade Federal do Espírito Santo, Jerônimo Monteiro - ES, email: racecilio@ yahoo.com.br.

${ }^{4}$ Engenheiro Agrônomo, D.Sc em Produção Vegetal, Professor Adjunto, Departamento de Ciências Florestais e da Madeira, Universidade Federal do Espírito Santo, Jerônimo Monteiro - ES, email: sszanetti@yahoo.com.br
} 


\begin{abstract}
The use of rationally water has become increasingly important in recent years. Evapotranspiration is an important variable in the hydrologic cycle in which their knowledge is essential for irrigation management. The objective of this study is to evaluate the performance of the methods Priestley-Taylor, Hargreaves-Samani, Thornthwaite and Camargo, compared with the Penman-Monteith, in the estimation of reference evapotranspiration in three municipalities in the Rio de Janeiro State. Climate variables were used for the period from 01 January 2011 to 31 December 2013 from automatic weather stations INMET in the cities of Paraty, Petrópolis and Campos dos Goytacazes. Daily and monthly time scale were evaluated using the methods. For the comparative analysis between them and the standard method was performed simple linear regression analysis and calculations of statistical indexes: Pearson's correlation coefficient (r), index of agreement of Willmott (d) confidence index of Camargo performance (c). In the studied scales, methods of Priestley-Taylor and Hargreaves-Samani outperformed the other methods evaluated. The Priestley-Taylor method was more reliable in the estimation of the reference evapotranspiration in Paraty and Campos dos Goytacazes, and Hargreaves-Samani method for Petrópolis.
\end{abstract}

Keywords: irrigation, Penman-Monteith, climate variables

\section{INTRODUÇÃO}

A disponibilidade de água no planeta tem diminuído nos últimos anos devido a uma combinação de frequentes secas e competição por recursos hídricos entre os usos agrícola, industrial e urbano (ORTEGA-FARIAS et al., 2009). A determinação precisa do consumo de água pelas culturas é fundamental para o uso racional da água (CAPORUSSO; ROLIM, 2015), no qual pode ser determinado através do cálculo do balanço hídrico no solo.

A evapotranspiração é um dos principais processos componentes do ciclo hidrológico e a principal componente no balanço hídrico do solo, no qual o seu conhecimento é essencial para um planejamento econômico no uso dos recursos hídricos (FANAYA JUNIOR et al., 2012); e serve de parâmetro para estudos de avaliação ambiental e de manejo de bacias hidrográficas (RAZIEI; PEREIRA, 2013).

A evapotranspiração é definida como a combinação de dois processos distintos: a evaporação que, é a água perdida pela superfície do solo; e a transpiração, que é a água perdida a partir da cultura (ALLEN et al., 1998). A medição direta da evapotranspiração é difícil e dispendiosa, requerendo equipamentos e pessoal especializados, portanto a sua determinação por meio de estimativas é a forma mais comum.
Existem diversos métodos para estimar evapotranspiração de referência $\left(\mathrm{ET}_{0}\right)$, no qual se destaca o método de Penman-Monteith (PM) (LÓPEZ URREA et al., 2006).

Alguns autores avaliaram o método PM comparando-o com lisímetros, e concluíram que esse é o método mais eficiente para estimar a $\mathrm{ET}_{0}$ (BARROS et al., 2009; SOUZA et al., 2011). Entretanto, este método necessita do conhecimento de vários elementos meteorológicos, que nem sempre se encontram disponíveis (MARTí et al., 2015). O uso de equações simplificadas é uma alternativa potencial para estimar a $\mathrm{ET}_{0}$ (ALENCAR et al., 2015; CARVALHO et al., 2015). Desta forma, há a necessidade de estudos para que se determine o melhor método empírico em cada local (TABARI, 2010; TAGLIAFERRE et al., 2010; FRANÇA NETO et al., 2011; BEZERRA et al., 2014; ALENCAR et al., 2015).

Desta forma, objetivou-se avaliar o desempenho dos métodos empíricos de Priestley-Taylor, Hargreaves-Samani, Thornthwaite \& Camargo, em comparação com o método de PM, na estimação da evapotranspiração de referência em três municípios do Estado do Rio de Janeiro.

\section{MATERIAL E MÉTODOS}




\section{MÉTODOS EMPÍRICOS PARA ESTIMATIVA DA EVAPOTRANSPIRAÇÃO DE REFERÊNCIA NO ESTADO DO RIO DE JANEIRO}

O presente trabalho foi realizado utilizando dados das estações meteorológicas automáticas do Instituto Nacional de Meteorologia (INMET), dos municípios de
Paraty, Petrópolis e Campos dos Goytacazes, situados respectivamente nas regiões $\mathrm{Sul}$, Serrana e Norte do Estado do Rio de Janeiro, conforme a Figura 1.
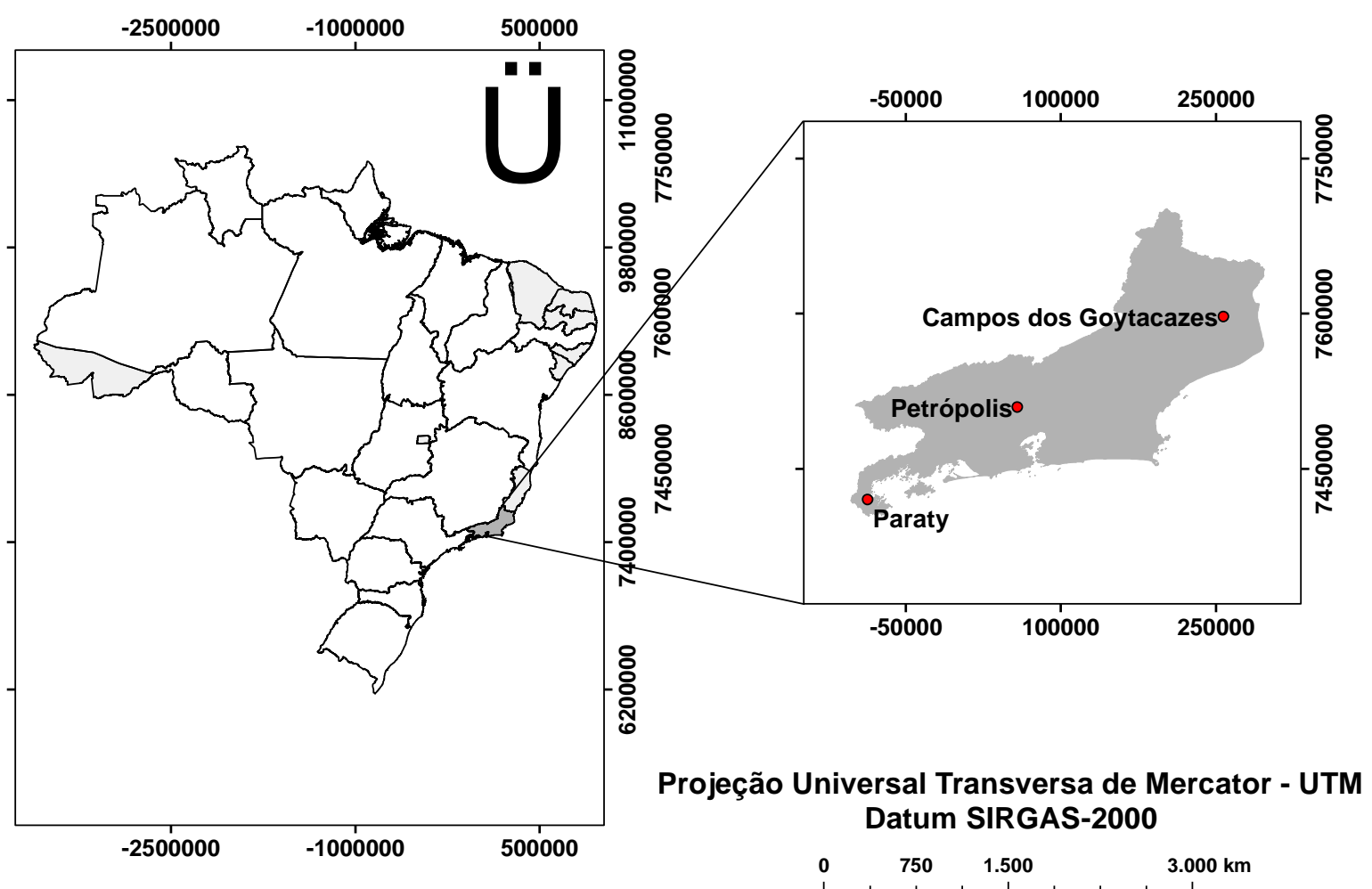

Figura 1. Localização das estações meteorológicas do INMET utilizadas no estudo.

Para a realização deste trabalho foram utilizadas as seguintes variáveis: temperatura do ar $\left({ }^{\circ} \mathrm{C}\right)$; umidade relativa do ar $(\%)$, velocidade do vento a $2 \mathrm{~m}$ de altura $\left(\mathrm{m} \mathrm{s}^{-1}\right) \mathrm{e}$ radiação solar global $\left(\mathrm{MJ} \mathrm{m}^{-2} \mathrm{dia}^{-1}\right)$, referentes ao período de 01 de janeiro de 2011 até 31 de dezembro de 2013, dos municípios em estudo. Os municípios nos quais as estações estão localizadas, suas respectivas latitude, longitude, altitude e período utilizado nesse estudo, estão descritos na Tabela 1. De acordo com a classificação de Köppen, o clima predominante de Paraty é o Tropical sem estação seca (Af), Petrópolis tem clima do tipo Subtropical úmido sem estação seca $(\mathrm{Cfb})$ e Campos dos Goytacazes é classificado como do tipo Tropical com inverno seco (Aw) (ALVARES et al., 2013).

Tabela 1. Nome da estação, código, localização geográfica, altitude e período utilizado das estações meteorológicas avaliadas nesse estudo.

\begin{tabular}{cccccc}
\hline Estação & Código & Latitude $\left(^{\circ}\right)$ & Longitude $\left(^{\circ}\right)$ & Altitude (m) & Período \\
\hline Paraty & A619 & $-23,22$ & $-44,72$ & 4 & $2011-2013$ \\
Petrópolis & A610 & $-22,45$ & $-43,28$ & 1777 & $2011-2013$ \\
Campos dos Goytacazes & A607 & $-21,70$ & $-41,35$ & 25 & $2011-2013$ \\
\hline
\end{tabular}

Os métodos utilizados para estimar a $\mathrm{ET}_{0}$ foram: Priestley-Taylor, HargreavesSamani, Thornthwaite e Camargo. Todos foram comparados ao método de Penman-Monteith, considerado padrão pela FAO. 
Método de Penman-Monteith FAO 56 (PM)

A equação padrão de Penman-Monteith FAO-56 (PM) (Allen et al., 1998) foi aplicada para estimar a $\mathrm{ET}_{0}$, cujos valores foram utilizados como referência. $\mathrm{A} \mathrm{ET}_{0} \mathrm{PM}$ diária foi calculada pela Equação1:

$$
E T_{0}{ }^{P M}=\frac{0,408 \cdot \Delta \cdot(R n-G)+\gamma \cdot \frac{900}{(T+273)} \cdot U_{2} \cdot(e s-e a)}{\Delta+\gamma\left(1+0,34 \cdot U_{2}\right)}
$$

em que:

$\mathrm{ET}_{0}{ }^{\mathrm{PM}}=$ evapotranspiração de referência calculada pelo método de PM (mm dia $\left.{ }^{-1}\right) ; \Delta=$ inclinação da curva de saturação do vapor da água $\left(\mathrm{kPa}^{\circ} \mathrm{C}^{-1}\right) ; \mathrm{Rn}=$ radiação solar líquida $(\mathrm{MJ}$ $\left.\mathrm{m}^{-2} \mathrm{dia}^{-1}\right) ; \mathrm{G}=$ fluxo de calor no solo $\left(\mathrm{MJ} \mathrm{m}^{-2}\right.$ $\left.\mathrm{dia}^{-1}\right) ; \gamma=$ constante psicrométrica $\left(\mathrm{kPa}^{\circ} \mathrm{C}^{-1}\right) ; \mathrm{U}_{2}$ $=$ velocidade média do vento a $2 \mathrm{~m}$ de altura $(\mathrm{m}$ $\mathrm{s}^{-1}$ ); es = pressão de saturação do vapor d'água no ar $(\mathrm{kPa})$; ea $=$ pressão real do vapor d'água no ar $(\mathrm{kPa})$.

As variáveis da equação $\operatorname{PM}(\Delta, \mathrm{Rn}, \mathrm{G}, \gamma$, es, ea) foram calculadas conforme Allen et al. (1998), e considerou-se o albedo da grama de 0,23 .

\section{Método de Priestley-Taylor (1972) (PT)}

Os valores de evapotranspiração estimados pelo método de Priestley-Taylor foram obtidos pela Equação 2.

$$
\mathrm{ET}_{0}^{\mathrm{PT}}=\frac{\alpha \frac{\Delta+\gamma}{\gamma}(\mathrm{Rn}-\mathrm{G})}{\lambda}
$$

em que:

$\mathrm{ET}_{0}=$ evapotranspiração de referência diária calculada pelo método de PT (mm dia $\left.{ }^{-1}\right) ; \alpha=$ parâmetro de Priestley-Taylor, igual a 1,$26 ; \lambda=$ calor latente da água a $20^{\circ} \mathrm{C}, 2,45 \mathrm{MJ} \mathrm{kg}^{-1}$.

\section{Método de Hargreaves-Samani (1985) (HS)}

O método Hargreaves-Samani (1985) foi desenvolvido na Califórnia em condições semiáridas, necessitando apenas das temperaturas máxima e mínima, e da radiação solar extraterrestre, por intermédio da Equação 3.

$$
\mathrm{ET}_{0}{ }^{\mathrm{HS}}=0,0023 \cdot R a \cdot\left(T_{\max }-T_{\text {min }}\right)^{0,5} \cdot\left(T_{\text {med }}+17,8\right)
$$

em que:

$\mathrm{ET}_{0}{ }^{\mathrm{HS}}=$ evapotranspiração de referência calculada pelo método de Hargreaves-Samani $\left(\mathrm{mm} \mathrm{d}^{-1}\right) ; \mathrm{T}_{\max }=$ temperatura máxima do ar diária $\left({ }^{\circ} \mathrm{C}\right) ; \mathrm{T}_{\min }=$ temperatura mínima do ar

$$
\begin{gathered}
\mathrm{ET}_{0}{ }^{T H}=16\left(\frac{10 . \mathrm{T}}{\mathrm{I}}\right)^{\mathrm{a}}, \\
\operatorname{para} 0^{\circ} \mathrm{C} \leq \mathrm{T}<26,5^{\circ} \mathrm{C} \\
\mathrm{ET}_{0}{ }^{T H}=-415,85+32,24 \mathrm{~T}-0,43 \mathrm{~T}^{2}, \\
\text { para } \mathrm{T} \geq 26,5^{\circ} \mathrm{C}
\end{gathered}
$$$$
\left(\mathrm{mm} \mathrm{d}^{-1}\right) \text {. }
$$

\section{Método de Thornthwaite (1948) (TH)}

em que:

$\mathrm{T}=$ Temperatura média mensal $\left({ }^{\circ} \mathrm{C}\right)$;

$$
\mathrm{I}=\sum_{1}^{12}(0,2 T)^{1,514}
$$

$$
a=6,75 \cdot 10^{-7} I^{3}-7,71 \cdot 10^{-5} I^{2}+0,01791 I+0,49239
$$




\section{MÉTODOS EMPÍRICOS PARA ESTIMATIVA DA EVAPOTRANSPIRAÇÃO DE REFERÊNCIA NO ESTADO DO RIO DE JANEIRO}

A evapotranspiração diária foi obtida dividindo a $\mathrm{ET}_{0}$ pelo número de dias do mês, como mostra a Equação 8.

$$
\mathrm{ET}_{0}{ }^{T H}=\frac{E T_{0} \text { mensal }}{\mathrm{ND}}
$$

em que:

$\mathrm{ND}=$ número de dias do mês.

\section{Método de Camargo (1971) (CA)}

É um método empírico baseado no método de Thornthwaite. A evapotranspiração diária pelo método de Camargo foi obtida pela Equação 9.

$$
\mathrm{ET}_{0}{ }^{C A}=0,01 . \text { Ra.T.ND }
$$

\section{Análise dos dados}

Para a análise estatística do desempenho dos métodos empíricos estudados, foi calculada correlação entre os valores estimados pelos métodos empíricos e os valores obtidos pelo método padrão de Penman-Monteith - FAO 56. Para isso, utilizaram-se os indicadores estatísticos propostos por Camargo e Sentelhas (1997), que são: o coeficiente de correlação de Pearson (r); o índice de concordância de Willmott (d); e o índice de confiança de Camargo (c), e o coeficiente de determinação $\left(r^{2}\right)$ da regressão linear simples entre as variáveis. Avaliaram-se os métodos nas escalas diária e mensal.

$$
\mathrm{r}=\frac{\sum(\mathrm{xi}-\overline{\mathrm{x}})(\mathrm{yi}-\overline{\mathrm{y}})}{\sqrt{\left(\sum(\mathrm{xi}-\overline{\mathrm{x}})^{2}\right)\left(\sum(\mathrm{yi}-\overline{\mathrm{y}})^{2}\right)}}
$$

Em que:

$\mathrm{r}=$ coeficiente de correlação; $\mathrm{xi}$ = valores diários da $\mathrm{ET}_{0}$ obtidos pelo método padrão; e yi = valores diários da $\mathrm{ET}_{0}$ estimados pelos métodos empíricos.

$$
\mathrm{d}=1-\frac{\sum_{\mathrm{i}=1}^{\mathrm{n}}(\mathrm{yi}-\mathrm{xi})^{2}}{\sum_{\mathrm{i}=1}^{\mathrm{n}}(|\mathrm{yi}-\overline{\mathrm{y}}|+|\mathrm{xi}-\overline{\mathrm{x}}|)^{2}}
$$

em que:

$\mathrm{d}$ = índice de concordância ou ajuste; yi = evapotranspiração de referência obtida pelo método a ser testado, $\mathrm{mm} \mathrm{dia}^{-1}$; $\mathrm{xi}=$ evapotranspiração de referência obtida pelo método padrão, $\mathrm{mm} \mathrm{dia}^{-1} ; \overline{\mathrm{x}}=$ média dos valores obtidos pelo método padrão, $\mathrm{mm} \mathrm{dia}^{-1}$; em $=$ número de observações.

$$
\mathrm{c}=\mathrm{r} . \mathrm{d}
$$

Em que:

$\mathrm{c}=$ índice de confiança de Camargo.

A Tabela 2 representa a classificação apresentada por Camargo e Sentelhas (1997) para análise do índice c. A classificação do coeficiente de correlação, sugerida por Silva e Barros Filho (2003), está na Tabela 3.

Tabela 2. Critério para análise do desempenho dos métodos empíricos pelo índice c.

\begin{tabular}{cc}
\hline Índice c & Desempenho \\
\hline$>0,85$ & Ótimo \\
0,76 a 0,85 & Muito Bom \\
0,66 a 0,75 & Bom \\
0,61 a 0,65 & Mediano \\
0,51 a 0,60 & Sofrível \\
0,41 a 0,50 & Mau \\
$\leq 0,40$ & Péssimo \\
\hline
\end{tabular}

Fonte: Camargo e Sentelhas (1997).

Tabela 3. Critério para análise dos valores do coeficiente de correlação de Pearson (r).

\section{Valores do coeficiente $(r)$}

Classificação da correlação

Perfeita Positiva 


\begin{tabular}{cc}
0,70 a 0,99 & Muito Forte Positiva \\
0,30 a 0,69 & Moderada Positiva \\
0,01 a 0,29 & Fraca Positiva \\
0 & Nenhuma \\
$-0,01$ a $-0,29$ & Fraca Negativa \\
$-0,30$ a $-0,69$ & Moderada Negativa \\
$-0,70$ a $-0,99$ & Muito Forte Negativa \\
-1 & Perfeita Negativa \\
\hline
\end{tabular}

Fonte: Silva e Barros Filho (2003).

\section{RESULTADOS E DISCUSSÕES}

A análise comparativa entre os valores de evapotranspiração de referência, obtidos pelos métodos empíricos estudados e os valores estimados pelo método de Penman-Monteith, nas escalas estudadas, em Paraty-RJ, Petrópolis-RJ e Campos dos Goytacazes-RJ, encontram-se na Tabela 4.

Tabela 4. Parâmetros da equação de regressão $\left(\beta_{0}, \beta_{1}\right)$, coeficiente de determinação $\left(\mathrm{r}^{2}\right)$, coeficiente de correlação (r), índice de concordância (d), índice de confiança ou desempenho (c) nas escalas diária e mensal, em Paraty-RJ, Petrópolis-RJ e Campos dos Goytacazes-RJ.

\begin{tabular}{|c|c|c|c|c|c|c|c|c|c|}
\hline Localidade & Período & Métodos & $\boldsymbol{\beta}_{0}$ & $\beta_{1}$ & $\mathbf{r}^{2}$ & $\mathbf{r}$ & d & c & Classificação \\
\hline \multirow{4}{*}{ Paraty } & \multirow{4}{*}{ Diário } & $\mathrm{PT}$ & $-0,2$ & 1,09 & 0,90 & 0,95 & 0,98 & 0,93 & Ótimo \\
\hline & & HS & 1,27 & 0,75 & 0,73 & 0,86 & 0,90 & 0,77 & Muito Bom \\
\hline & & $\mathrm{TH}$ & 1,19 & 0,54 & 0,44 & 0,67 & 0,77 & 0,51 & Sofrível \\
\hline & & CA & 1,48 & 0,47 & 0,45 & 0,67 & 0,76 & 0,50 & Mau \\
\hline \multirow{4}{*}{ Petrópolis } & \multirow{4}{*}{ Diário } & $\mathrm{PT}$ & 0,79 & 0,75 & 0,68 & 0,82 & 0,9 & 0,75 & Bom \\
\hline & & HS & 0,71 & 0,65 & 0,77 & 0,88 & 0,9 & 0,79 & Muito Bom \\
\hline & & $\mathrm{TH}$ & 1,14 & 0,25 & 0,42 & 0,65 & 0,59 & 0,38 & Péssimo \\
\hline & & $\mathrm{CA}$ & 1,12 & 0,27 & 0,29 & 0,54 & 0,58 & 0,31 & Péssimo \\
\hline \multirow{4}{*}{$\begin{array}{l}\text { Campos dos } \\
\text { Goytacazes }\end{array}$} & \multirow{4}{*}{ Diário } & PT & 0,16 & 0,84 & 0,83 & 0,91 & 0,92 & 0,83 & Muito Bom \\
\hline & & $\mathrm{HS}$ & 1,32 & 0,59 & 0,78 & 0,88 & 0,84 & 0,74 & Bom \\
\hline & & $\mathrm{TH}$ & 1,28 & 0,44 & 0,55 & 0,74 & 0,7 & 0,52 & Sofrível \\
\hline & & $\mathrm{CA}$ & 1,59 & 0,36 & 0,44 & 0,67 & 0,62 & 0,41 & Mau \\
\hline \multirow{4}{*}{ Paraty } & \multirow{4}{*}{ Mensal } & PT & 1,18 & $-16,59$ & 0,94 & 0,97 & 0,97 & 0,94 & Ótimo \\
\hline & & HS & 1,03 & 7,33 & 0,9 & 0,95 & 0,93 & 0,88 & Ótimo \\
\hline & & TH & 0,94 & $-4,97$ & 0,71 & 0,85 & 0,88 & 0,74 & Bom \\
\hline & & CA & 1,00 & $-10,09$ & 0,86 & 0,93 & 0,93 & 0,86 & Ótimo \\
\hline \multirow{4}{*}{ Petrópolis } & \multirow{4}{*}{ Mensal } & PT & 0,98 & 4,17 & 0,67 & 0,82 & 0,89 & 0,73 & Bom \\
\hline & & HS & 0,91 & $-1,57$ & 0,79 & 0,89 & 0,90 & 0,80 & Muito Bom \\
\hline & & TH & 0,33 & 27,46 & 0,42 & 0,65 & 0,52 & 0,34 & Péssimo \\
\hline & & $\mathrm{CA}$ & 0,55 & 9,66 & 0,38 & 0,62 & 0,56 & 0,35 & Péssimo \\
\hline \multirow{4}{*}{$\begin{array}{c}\text { Campos dos } \\
\text { Goytacazes }\end{array}$} & \multirow{4}{*}{ Mensal } & $\mathrm{PT}$ & 0,97 & $-13,66$ & 0,89 & 0,94 & 0,91 & 0,86 & Ótimo \\
\hline & & HS & 0,72 & 22,09 & 0,85 & 0,92 & 0,88 & 0,81 & Muito Bom \\
\hline & & TH & 0,60 & 17,59 & 0,65 & 0,81 & 0,65 & 0,52 & Sofrível \\
\hline & & $\mathrm{CA}$ & 0,66 & 6,79 & 0,69 & 0,83 & 0,66 & 0,55 & Sofrível \\
\hline
\end{tabular}


Os resultados obtidos na escala diária para Paraty-RJ mostram que o método de PriestleyTaylor apresentou o melhor índice de desempenho $(c=0,93)$, sendo classificado como ótimo. Com um desempenho um pouco inferior, porém classificado como muito bom, está o método de Hargreaves-Samani. Para a estimação da $\mathrm{ET}_{0}$ na escala mensal em ParatyRJ, apenas o método de Thornthwaite foi classificado como bom. Os outros três métodos estudados, Priestley-Taylor, HargreavesSamani e Camargo, foram classificados como ótimos. Esses resultados demonstram a viabilidade no uso de qualquer um dos métodos estudados, na estimação da $\mathrm{ET}_{0}$ em escala mensal em Paraty-RJ.

Para as condições meteorológicas da localidade de Petrópolis-RJ, na escala diária, o método de Hargreaves-Samani foi o que apresentou o melhor desempenho, caracterizado como muito bom. O método de Priestley-Taylor, com um índice c igual a 0,745, também obteve um desempenho satisfatório, resultado semelhante ao encontrado por Reis et al. (2007) em Venda Nova do Imigrante - ES, que possui clima semelhante ao de Petrópolis-RJ (ALVARES et al., 2013).

França Neto et al. (2011), comparando os métodos na estimativa da evapotranspiração de referência em regiões produtoras de café brasileiras, encontrou um $\mathrm{r}^{2}$ de 0,72, para o método de HS em Vitória da Conquista - BA, na escala diária. $\mathrm{O}$ valor encontrado pelos autores aproxima-se do encontrado em Petrópolis-RJ, corroborando com este trabalho. Para a escala mensal em Petrópolis-RJ, observa-se que os métodos de Priestley-Taylor e HargreavesSamani apresentaram os melhores desempenhos, com índices c de 0,73 e 0,80, respectivamente.

Para Campos dos Goytacazes, os modelos de Priestley-Taylor e Hargreaves-Samani apresentaram os melhores desempenhos nas escalas avaliadas, resultado semelhante ao encontrado por Tagliaferre et al. (2010) em Eunápolis - BA. O método de Camargo obteve o maior erro da estimação da $\mathrm{ET}_{0}$, seguido do método de Thornthwaite. Fanaya Junior et al. (2012) avaliaram alguns métodos empíricos para estimar a $\mathrm{ET}_{0}$ em Aquidauana - MS, sendo que os métodos de PT e HS também obtiveram bons desempenhos nas escala diária e mensal, corroborando com este trabalho. A similaridade dos resultados pode ser explicada, provavelmente, devido a Aquidauana possuir o mesmo tipo de clima (Aw) que Campos dos Goytacazes (ALVARES et al., 2013).

O comportamento da tendência e da magnitude da $\mathrm{ET}_{0}$ em Paraty-RJ, Petrópolis-RJ e Campos dos Goytacazes-RJ, durante o período de estudo, na escala mensal, entre os métodos avaliados e o método de Penman-Monteith FAO 56 está representado na Figura 2. Em Paraty-RJ, nota-se que os modelos de PriestleyTaylor e Hargreaves-Samani apresentaram maior similaridade com o método de PenmanMonteith. Em relação à magnitude, levando em conta a diferença média mensal, o modelo de Priestley-Taylor mostrou a maior similaridade com PM, onde superestimou a $\mathrm{ET}_{0}$ em 2,4 mm. $\mathrm{O}$ método de Hargreaves-Samani foi o que mais superestimou a $\mathrm{ET}_{0}$ nesta cidade, sendo este resultado similar ao encontrado por Carvalho et al. (2015). O modelo de Camargo mostrou um melhor desempenho nos meses mais chuvosos do ano na região (outubro à março), já o modelo de Hargreaves-Samani apresentou boas estimativas no período seco do ano (abril à setembro).

Em relação à magnitude, em PetrópolisRJ, os métodos de Priestley-Taylor e Hargreaves-Samani acompanharam, de uma forma geral, os valores estimados por Penman. Na média, o método de Priestley-Taylor obteve maior similaridade com PM, superestimando a $\mathrm{ET}_{0}$ em 2,4 mm por mês, já o método de Thornthwaite teve o pior resultado, subestimando a $\mathrm{ET}_{0} \mathrm{em} 30,6 \mathrm{~mm}$. O método de Hargreaves-Samani obteve melhor desempenho no período chuvoso na região. Palaretti et al. (2014) obtiveram resultados semelhantes para o método de Hargreaves-Samani nos municípios de Itapetininga-SP e Botucatu-SP, haja vista que estas cidades possuem clima semelhante ao de Petrópolis (ALVARES et al., 2013). Porém, para os autores, o método de HS apresentou tendência de superestimar a $\mathrm{ET}_{0}$, ao contrário do encontrado em Petrópolis-RJ. 
Vallory et al.

$\mathrm{Na}$ análise do comportamento da tendência e da magnitude da $\mathrm{ET}_{0}$, na escala mensal, durante o período de estudo em Campos dos Goytacazes-RJ, observa-se que todos os métodos avaliados tendem a subestimar os valores da $\mathrm{ET}_{0}$ de Penman-Monteith. Na média, o método de Camargo foi o que mais subestimou os valores da $\mathrm{ET}_{0}$, e o método de HargreavesSamani foi o que menos subestimou, cerca de $16,2 \mathrm{~mm}$.

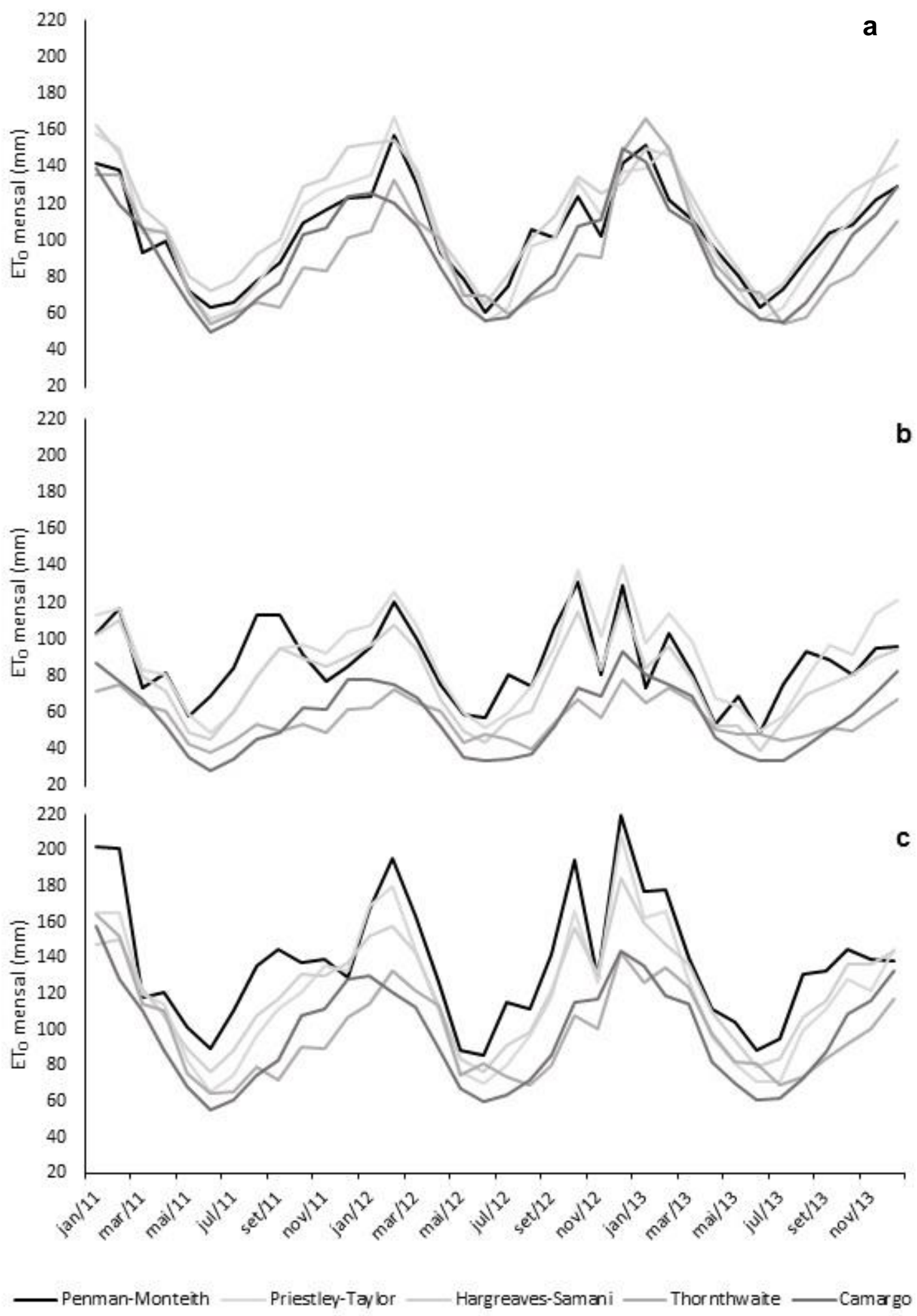

Figura 2. Evolução da evapotranspiração de referência para cada mês do período estudado, de todos os métodos avaliados, nas localidades de Paraty-RJ (a), Petrópolis-RJ (b) e Campos dos Goytacazes-RJ (c). 


\section{CONCLUSÕES}

Para as localidades de Paraty e Campos dos Goytacazes, a utilização do método de Priestley-Taylor apresentou boa confiabilidade na estimação da evapotranspiração de referência nas escalas mensal e diária. Para Petrópolis, o método mais indicado é o de HargreavesSamani nas escalas analisadas.

Não se recomenda a utilização dos métodos de Thornthwaite na escala mensal no inverno, e de Camargo na escala diária no verão nas três localidades estudadas.

\section{REFERÊNCIAS}

ALENCAR， L. P.; SEDIYAMA， G. C.; MANTOVANI, E. C. Estimativa da evapotranspiração de referência (ETo padrão FAO), para Minas Gerais, na ausência de alguns dados climáticos. Engenharia Agrícola, v. 35, n. 1, p. 39-50, 2015.

ALLEN, R. G. A. Penman for all seasons. Journal of Irrigation and Drainage Engineering, v. 112, n. 4, p. 348-386, 1986.

ALLEN, R. G.; PEREIRA, L. S.; RAES, D.; SMITH, M. Crop evapotranspiration: guidelines for computing crop water requirements. Rome: FAO, 1998. 300 p. (Irrigation and Drainage Paper, 56).

ALVARES, C. A.; STAPE, J. L.; SENTELHAS, P. C.; GONÇALVES, J. L. M.; SPAROVEK, G. Köppen's climate classification map for Brazil. Meteorologische Zeitschrift, v. 22, n. 6, p. 711-728, 2013.

BARROS, V. R.; SOUZA, A. P.; FONSECA, D. F.; SILVA, L. B. D. Avaliação da evapotranspiração de referência na região de Seropédica, Rio de Janeiro, utilizando lisímetro de pesagem e modelos matemáticos. Revista Brasileira de Ciências Agrárias, v. 4, p. 198203, 2009.

BEZERRA, J. M.; MOURA, G. B. A.; SILVA, E. F. F.; LOPES, P. M. O.; SILVA, B. B.
Estimativa da evapotranspiração de referência diária para Mossoró (RN, Brasil). Revista Caatinga, v. 27, n. 3, p. 211-220, 2014.

CAMARGO, A. P; SENTELHAS, P. C. Avaliação do desempenho de diferentes métodos de estimativas da evapotranspiração potencial no Estado de São Paulo, Brasil. Revista Brasileira de Agrometeorologia, v. 5, n. 1, p. 8997, 1997.

CAPORUSSO, N. B.; ROLIM, G. de S. Reference evapotranspiration models using different time scales in the Jaboticabal region of São Paulo, Brazil. Acta Scientiarum. Agronomy (Impresso), v. 37, p. 1-9, 2015.

CARVALHO, D. F.; ROCHA, H. S.; BONOMO, R.; SOUZA, A. P. Estimativa da evapotranspiração de referência a partir de dados meteorológicos limitados. Pesquisa Agropecuária Brasileira, v. 50, n. 1, p. 1-11, 2015.

FANAYA JÚNIOR, E. D.; LOPES, A. S.; OLIVEIRA, G. Q.; JUNG, L. H. Métodos empíricos para estimativa da evapotranspiração de referência para Aquidauana, MS. Irriga, v. 17, n. 4, p. 418-434, 2012.

FRANÇA NETO, A.C.; MANTOVANI, E. C.; VICENTE, M. R.; VIEIRA, G. H. S.; SEDIYAMA, G. C.; LEAL, B. G. Comparação entre métodos simplificados de estimativa da evapotranspiração de referência $\left(\mathrm{ET}_{0}\right)$ para regiões produtoras de café brasileiras. Coffee Science, v. 6, n. 2, p. 159-171, 2011.

HARGREAVES, G. H.; SAMANI, Z. A. Reference crop evapotranspiration from ambient air temperature. Applied Engineering in Agriculture, v. 1, n. 2, p. 96-99, 1985.

LÓPEZ-URREA, R.; MARTIN DE SANTA OLALLA, F.; FABEIRO, C.; MORATALLA, A. Testing evapotranspiration equations using lysimeter observations in a semiarid climate. Agricultural Water Management, v. 85, n.1, p. 15-26, 2006. 
MARTÍ, P.; ZARZO, M.; VANDERLINDEN, K.; GIRONA, J. Parametric expressions for the adjusted Hargreaves coefficient in Eastern Spain. Journal of Hydrology, v. 529, n. 1, p. 1713-1724, 2015.

ORTEGA-FARIAS, S.; IRMAK, S.; CUENGA, R. H. Special issue on evapotranspiration measurement and modeling. Irrigation Science, v. 28, n. 1, p. 1-3, 2009.

PALARETTI, L. F.; MANTOVANI, E. C.; SEDIYAMA, G. C. Comparação entre métodos de estimativa da evapotranspiração de referência $\left(\mathrm{ET}_{0}\right)$ em regiões citrícolas paulistas. Engenharia Agrícola, v. 34, n. 1, p. 38-47, 2014.

RAZIEI, T.; PEREIRA, L. S. Estimation of ETo with Hargreaves-Samani and FAOPM temperature methods for a wide range of climates in Iran. Agricultural Water Management, v. 121, n. 4, p. 1-18, 2013.

REIS, E. F.; BRAGANÇA, R.; GARCIA, G. O.; PEZZOPANE, J. E. M.; TAGLIAFERRE, C.
Estudo comparativo da estimativa da evapotranspiração de referência para três localidades do estado do Espírito Santo no período seco. Idesia, v. 25, n. 3, p. 75-84, 2007.

SILVA, D.; BARROS FILHO, J. 2003. Estatística. São Paulo, USP. (Apostila).

SOUZA, A. P. de; CARVALHO, D. F. de; SILVA, L. B. D. da; ALMEIDA, F. T. de; ROCHA, H. S. da. Estimativas da evapotranspiração de referência em diferentes condições de nebulosidade. Pesquisa Agropecuária Brasileira, v. 46, n. 3, p. 219228, 2011.

TABARI, H. Evaluation of reference crop evaporanspiration equations in various climates. Water Resource Management, v. 24, n. 10, p. 2311-2337, 2010.

TAGLIAFERRE, C.; SILVA, R.; ROCHA, F.; SANTOS, L. Estudo Comparativo de diferentes metodologias para determinação da evapotranspiração de referência em EunápolisBA. Revista Caatinga, v. 23, n. 1, p. 103-111, 2010. 